available, together responsible for 36 area under the concentration time curves (AUCs) and trough concentrations. First, hematocrit-corrected concentrations were derived using a formula describing the relationship between whole blood concentrations, hematocrit, and plasma concentrations. Subsequently, target exposure was evaluated using the converted plasma target concentrations. Ultimately, differences in interpretation of target exposure were identified and evaluated.

Results In total, 92\% of our patients had a lower hematocrit (median 0.29) than the reference value of adult renal transplant patients. A different evaluation of target exposure for either trough level, AUC or both, was defined in $42 \%$ of our patients, when applying hematocrit corrected concentrations.

Conclusion A critical role for hematocrit in therapeutic drug monitoring of tacrolimus in pediatric kidney transplant patients is suggested in this study. Therefore, we believe that hematocrit correction could be a step towards improvement of tacrolimus dose individualization.

Disclosure(s) All authors declare that they have no conflict of interest. This work was recently accepted for publication in Pediatric Nephrology. We are aware of the statement in the guideline about previously published work, however as we believe that this work suits the aim and topics of the congress very well, we chose to submit this work and will ofcourse leave the final decision to you.

\section{P89 EMICIZUMAB DEVELOPMENT IN PAEDIATRIC PATIENTS WITH CONGENITAL HEMOPHILIA A AND INHIBITORS AGAINST FACTOR VIII}

${ }^{1} \mathrm{C}$ Schmitt* ${ }^{2} \mathrm{~K}$ Yoneyama, ${ }^{3} \mathrm{~T}$ Chang, ${ }^{4} \mathrm{~S}$ Retout, ${ }^{5} \mathrm{H}-\mathrm{P}$ Grimm, ${ }^{3} \mathrm{GG}$ Levy. ${ }^{1} \mathrm{~F}$. Hoffmann-La Roche Ltd, Basel, Switzerland; ${ }^{2}$ Chugai Pharmaceutical, Tokyo, Japan; ${ }^{3}$ Genentech, San Francisco, CA, USA; ${ }^{4}$ F. Hoffmann-La Roche Ltd, Paris, France; ${ }^{5}$ F Hoffmann-La Roche Ltd, Basel, Switzerland

\subsection{6/archdischild-2019-esdppp.127}

Background Emicizumab, a bispecific monoclonal antibody, bridges activated factor (F) IX (FIXa) and FX, restoring missing FVIIIa function in patients with hemophilia A (PwHA). It has been recently approved for routine prophylaxis of bleeding episodes in PwHA with FVIII inhibitors in all age groups. We herein describe the paediatric development of emicizumab with a focus on dose selection, study design and extrapolation of efficacy.

Methods The dosing regimen for the HAVEN 1 study (adolescents/adults) was selected based on the exposure-response (annualized bleeding rate $[\mathrm{ABR}]$ ) relationship derived from a phase I/II study in adolescent/adult PwHA. Pharmacokinetic (PK) simulations, with or without maturation of clearance (CL MAT), were performed to guide selection of the dosing regimen for the HAVEN 2 study (paediatrics) aiming to achieve the same target exposure as in HAVEN 1. HAVEN 2 employed a flexible design with possibility for individual- and population-level dose up-titration. Additional PK modeling and simulations together with exposure-response analyses were used to support full extrapolation of efficacy in any age category where no data was available.

Results $1.5 \mathrm{mg} / \mathrm{kg} /$ week in HAVEN 1 provided mean trough concentrations of $\sim 50 \mu \mathrm{g} / \mathrm{mL}$ and was associated with a $87 \%$ reduction in ABR. Doses of $\geq 1.5$ (with CL MAT) and $\geq$
$2.25 \mathrm{mg} / \mathrm{kg} /$ week (without CL MAT) were predicted to achieve this target exposure in children. HAVEN 2 began by investigating the same dose as HAVEN 1. Similar trough concentrations of $\sim 50 \mu \mathrm{g} / \mathrm{mL}$ were achieved in children and were associated with low ABR. No patients $<1$ year were enrolled in HAVEN 2. PK simulations and pharmacological rationale suggested that a different dose for these patients was not warranted.

Conclusions Efficacy of emicizumab in adolescents/adults was demonstrated in HAVEN 1, partially extrapolated to HAVEN 2 participants 1-11 year of age, and fully extrapolated in patients $<1$ year.

Disclosure(s) Authors are Roche/Genentech employees

\section{P90 NEUROPSYCHIATRIC DISORDER AND MONTELUKAST: A CASE REPORT AND VIGIBASE ${ }^{\circledR}$ ANALYSIS}

1,2 Scholz*, 1,35 Banholzer, ${ }^{1,3} \mathrm{M}$ Haschke. ${ }^{1}$ Clinical Pharmacology and Toxicology, Department of General Internal Medicine, Inselspital Bern, Institute of Pharmacology, University of Bern; ${ }^{2}$ Institute of Pharmacology, University of Bern; ${ }^{3}$ Institute of Pharmacology, University of Bern, Switzerland, Bern, Switzerland

\subsection{6/archdischild-2019-esdppp.128}

Learning objective Recognize neuropsychiatric symptoms as possible adverse drug reactions (ADR) associated with the leukotriene receptor antagonist Montelukast in children

Clinical Case An 11-year-old boy suffering from asthma presented to his pediatrician with an acute onset of nervousness, restlessness and irritability. The teacher noticed a decline in school performance with a reduced attention span. The patient had been treated with Salbutamol (Ventolin ${ }^{\circledR}$ ) and Salmeterol/ Fluticasone (Seretide ${ }^{\circledR}$ ) for the last few years. A treatment with Montelukast chewable tablets was started four months ago. The ADR was reported to the Regional Pharmacovigilance Centre (RPVC) Bern. The termination of the therapy with Montelukast lead to an amelioration of the symptoms. According to the WHO-UMC Causality Categories, ${ }^{1}$ the causality of Montelukast and the described symptoms was classified as 'probable'. The causality of the comedication was considered 'unlikely' as it had been used for several years without complications. After work-up at the RPVC, the case was reported anonymously to the National Pharmacovigilance Center of the Swiss Agency for Therapeutic Products Swissmedic.

Discussion Montelukast is a cysteinyl-leukotriene type 1-receptor antagonist used in the treatment of bronchial asthma in adults and children. Psychiatric disorders such as agitation, psychomotor hyperactivity (including irritability and restlessness), disorders of attention and memory impairment (and others) are listed as known ADRs of Montelukast. ${ }^{2} 3$ The WHO pharmacovigilance database VigiBase ${ }^{\circledR}$ lists a total of 20'897 ADR reports for Montelukast, of which 4'705 $(22.5 \%)$ refer to nervous system disorders and 6'828 (32.7\%) to psychiatric disorders. Within the group of nervous system disorders $256(5.4 \%)$ reports of psychomotor hyperactivity, $232(4.9 \%)$ reports of disturbance in attention and $91(1.9 \%)$ reports of memory impairment were recorded. ${ }^{4}$ The most common symptoms in the group of psychiatric disorders are depression (1'311, 19.2\%) and aggressive behavior (1'175, 17.2\%). If psychiatric ADRs occur, the risks and benefits of Montelukast should be reassessed. 